\title{
CRYSTAL PRECIPITATION AND DISSOLUTION IN A POROUS MEDIUM: EFFECTIVE EQUATIONS AND NUMERICAL EXPERIMENTS*
}

\author{
T.L. VAN NOORDEN ${ }^{\dagger}$
}

\begin{abstract}
We investigate a two-dimensional micro-scale model for crystal dissolution and precipitation in a porous medium. The model contains a free boundary and allows for changes in the pore volume. Using a level-set formulation of the free boundary, we apply a formal homogenization procedure to obtain upscaled equations. For general micro-scale geometries, the homogenized model that we obtain falls in the class of distributed microstructure models. For circular initial inclusions the distributed microstructure model reduces to system of partial differential equations coupled with an ordinary differential equation. In order to investigate how well the upscaled equations describe the behavior of the micro-scale model, we perform numerical computations for a test problem. The numerical simulations show that for the test problem the solution of the homogenized equations agrees very well with the averaged solution of the micro-scale model.
\end{abstract}

1. Introduction. In this paper we use a formal limiting procedure with asymptotic expansions to derive a macroscopic law for crystal dissolution and precipitation in a porous medium. The microscopic model that serves as the staring point for the limiting process, incorporates the change in volume of the pore space as a result of the precipitation/dissolution process. In [17], the same microscopic model is considered in a thin strip. In the present paper we investigate the model on a perforated domain.

Macroscopic laws for reactive transport in porous media, which include the present case of crystal dissolution and precipitation, are of practical importance in many physical, biological and chemical applications. Macroscopic laws for reactive transport in porous media are derived rigorously in, e.g., [6]. For the more specific case of crystal dissolution and precipitation, macroscopic models are given in $[3,4,7,8]$. In these papers the presented macroscopic models are analysed, but are not supported by a rigorous derivation. In most of these papers also the numerical solution of the proposed model equations is studied. Related work, in which the transport of dissolved material is analysed, can be found in $[14,16]$.

The main difficulty in performing the formal homogenization for the crystal dissolution and precipitation reaction is that the equations that describe the micro-scale processes contain a free boundary. This free boundary describes the interface between the layer of crystalline solid attached to the grains and the fluid occupying the pores. The location of this free boundary is an unknown in the model and moves with speed proportional to the local dissolution/precipitation rate. The micro-scale model with the free boundary has been studied in [18] in a one dimensional setting and without flow. Other works that study crystal dissolution and precipitation on the micro-scale are [12] and [13].

The crystal dissolution and precipitation problem has been studied also in [7] and $[15,19]$. The main difference between the cited papers and the present paper is that in

\footnotetext{
*Part of this research has been funded by the Dutch BSIK/BRICKS project.

${ }^{\dagger}$ Department of Mathematics and Computer Science, Technische Universiteit Eindhoven, P.O. Box 513, 5600 MB Eindhoven, The Netherlands, email: T.L.v.Noorden@tue.nl
} 


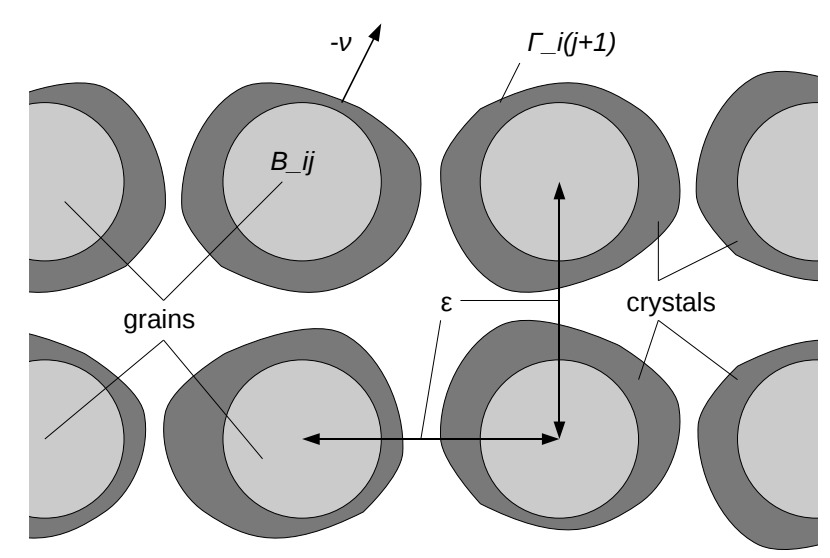

FIG. 2.1. Schematic representation of porous medium with layers of crystalline solid attached to the grains.

the cited papers it is assumed that the thickness of the layer of crystalline solid attached to the grains domain is negligible so that the pore geometry can assumed to be fixed. In this case the formal homogenization is a rather standard procedure and the macroscopic equations can be derived straightforwardly.

In this paper we do take into account the change in the pore volume due to the precipitation/dissolution reaction. For the micro-scale model this results in a free-boundary problem, and for the macroscopic model this results in a permeability and porosity that depend on the local pore geometry. The behaviour of solutions of the micro-scale model are numerically studied using the Arbitrary Lagrangian Eulerian method. The structure of this paper is as follows. In Section 2 we will discuss the model equations that describe the processes at the micro-scale. In Section 3, we will derive using a formal limiting argument the effective equations for the limit of $\epsilon$ to zero, and in Section 4 we show that the effective model can be simplified for certain initial configurations. In Section 5 the numerical results for both the micro-scale model and the effective equations are presented and compared.

2. Model equations. We consider the same model for crystal dissolution and precipitation as is described in [17]. In this paper, however, we consider a different micro-scale geometry of the porous medium. The porous medium is in the present paper represented by a two dimensional preforated domain. We denote the two dimensional bounded domain by $\Omega$, with boundary $\Gamma$, and we suppose that the grains of the porous medium are circles. Let the centers of the cicles $B_{i j}$, all with radius $R_{g}<\epsilon / 2$, be located in a equidistant grid with nodes at $(\epsilon i, \epsilon j)$, where $\epsilon$ is a small dimensionless length scale. The pore space in completely occupied by a fluid which contains dissolved solutes (ions) which can precipitate on the grain boundary and form a crystalline solid, the precipitate. The reverse reaction of dissolution is also possible. We only consider one solute (the cation), even though dissolution and precipitation involves another species, the anion. This is a simplified setting that is considered also in [17, 18], which can be achieved by considering another quantity, the total electric charge (see [7, 15]). Under appropriate boundary and initial conditions, the total charge remains constant in time and space, and the two species have equal concentrations. The boundary that is the interface between the crystalline solid that surrounds the circle $B_{i j}$ and the pore space is denoted by $\Gamma_{i j}(t)$, with 
normal $\nu^{\epsilon}$, pointing into the crystalline phase, and its normal velocity is denoted by $v_{n}^{\epsilon}$ (see Fig. 2.1). The dimensionless equation for the normal velocity is given by (see [17])

$$
v_{n}^{\epsilon}=-\epsilon k\left(r\left(u^{\epsilon}\right)-w^{\epsilon}\right), \quad \text { on } \Gamma_{i j}(t),
$$

where $k$ is the Damköhler number, $r$ is a rate function for the precipitation rate, and where $w^{\epsilon} \in H\left(\operatorname{dist}\left(x, B^{\epsilon}\right)\right)$, with $B^{\epsilon}:=\cup B_{i j}$, is a multivalued dissolution rate, with dist the Euclidian distance function, and with $H$ the set-valued Heaviside graph,

$$
H(u)= \begin{cases}\{0\}, & \text { if } u<0, \\ {[0,1],} & \text { if } u=0, \\ \{1\}, & \text { if } u>0\end{cases}
$$

In principle the equation (2.1) determines the evolution and thus the position of the free interfaces $\Gamma_{i j}(t)$. To apply formal homogenization, we can of coarse expand the unknowns in (2.1) in a power series in $\epsilon$, but it would be convenient not only to be able to expand the velocity of the interface, but also to be able to expand the position of the interface in a power series in $\epsilon$. The idea that we pursue in the present paper, is to describe the free boundaries $\Gamma_{i j}(t)$ with a level set function. A level set function is a convenient tool to deal with moving interfaces, and has also inspired new ways to solve numerically moving boundary problems $[9,10,11]$. We identify the interface with the zero-level set of the level set function, say $S^{\epsilon}$. The level set equation

$$
S_{t}^{\epsilon}+v_{n}^{\epsilon}\left|\nabla S^{\epsilon}\right|=0
$$

describes the evolution of the interface. We can now also expand the function $S$ in a series and use the machinery of formal homogenization.

It is important to note that we assume that the layers of crystalline solid surrounding the different grains do not touch each other, so that $\Gamma_{i j}(t) \cap \Gamma_{k l}(t)=\emptyset$ if $i \neq k$ or $j \neq l$, and we also assume that the grains do not intersect the outer boundary of the domain $\Omega$, so that $\Gamma \cap \Gamma_{i j}(t)=\emptyset$ for all $i, j$. We denote the solute concentration by $u^{\epsilon}$, the velocity of the fluid phase by $q^{\epsilon}$, the pressure by $p^{\epsilon}$. All these unknowns are dimensionless. Note that for the formulation of the equations we need a continuous extension of $u^{\epsilon}$ to the whole of $\Omega$, which is in equations below denoted by $\bar{u}^{\epsilon}$. The model is given by

$$
\begin{aligned}
& \left\{\begin{array}{l}
u_{t}^{\epsilon}=\nabla \cdot\left(D \nabla u^{\epsilon}-q^{\epsilon} u^{\epsilon}\right), \\
\epsilon^{2} \mu \Delta q^{\epsilon}=\nabla p^{\epsilon}, \\
\nabla \cdot q^{\epsilon}=0,
\end{array}\right. \\
& \left\{\begin{array}{l}
S_{t}^{\epsilon}=\epsilon k\left(r\left(\bar{u}^{\epsilon}\right)-w^{\epsilon}\right)\left|\nabla S^{\epsilon}\right|, \quad \text { in } \Omega^{\epsilon}(t), \\
w^{\epsilon} \in H\left(\operatorname{dist}\left(x, B^{\epsilon}\right)\right),
\end{array}\right. \\
& \left\{\begin{array}{l}
\nu^{\epsilon} \cdot\left(D \nabla u^{\epsilon}-q^{\epsilon} u^{\epsilon}\right)=-\epsilon k\left(r\left(u^{\epsilon}\right)-w^{\epsilon}\right)\left(\rho-u^{\epsilon}\right), \quad \text { on } \Gamma^{\epsilon}(t) \\
q^{\epsilon}=-\epsilon K k\left(r\left(u^{\epsilon}\right)-w^{\epsilon}\right) \nu^{\epsilon},
\end{array}\right. \\
& \left\{\begin{array}{l}
u^{\epsilon}(x, t)=u_{b}(x, t), \\
q^{\epsilon}(x, t)=q_{b}(x, t),
\end{array}\right. \\
& \left\{\begin{array}{l}
u^{\epsilon}(x, 0)=u_{I}(x), \\
S^{\epsilon}(x, 0)=S_{I}(x),
\end{array}\right.
\end{aligned}
$$


where

$$
\begin{aligned}
& \Omega^{\epsilon}(t):=\left\{x \in \Omega \mid S^{\epsilon}(x, t)<0\right\} \\
& \Gamma^{\epsilon}(t):=\left\{x \in \Omega \mid S^{\epsilon}(x, t)=0\right\}
\end{aligned}
$$

and where

$$
\nu^{\epsilon}=\frac{\nabla S^{\epsilon}}{\left|\nabla S^{\epsilon}\right|}
$$

denotes the normal on the boundary $\Gamma^{\epsilon}(t)$.

The model equations contain the positive parameters $D$, which is the dimensionless diffusion coefficient, $\mu$, which is the dimensionless viscosity, $k$, which is the Damköhler number, and $\rho$, which is the dimensionless density of the crystalline phase. The auxiliary function $w^{\epsilon}$ acts as the scaled dissolution rate and, when $\operatorname{dist}\left(x, B^{\epsilon}\right)>0, w^{\epsilon}$ attains the value 1 , and when $\operatorname{dist}\left(x, B^{\epsilon}\right)=0$, we have $w^{\epsilon}=r\left(u^{\epsilon}\right)$. With respect to the reaction rate function $r(u)$, we assume

1. $r: \mathbb{R} \rightarrow[0, \infty)$ is locally Lipschitz;

2. a unique $u_{-} \in[0, \rho)$ exists such that $r(u)=0$ for all $u \leq u_{-}$and $r(u)$ is strictly increasing if $u>u_{-}$.

3. a unique $u_{s} \in\left(u_{-}, \rho\right)$ exists such that $r\left(u_{s}\right)=1$.

The parameter $K$, which expresses volume changes due to precipitation or dissolution [17], may also be negative, and is between the bounds

$$
\frac{u_{s}-\rho}{u_{s}}<K<1
$$

Furthermore, we assume the following inequalities

$$
0 \leq u_{I}<\rho
$$

3. Formal homogenization. For the formal homogenization we assume the following formal asymptotic expansions for $u^{\epsilon}, S^{\epsilon}, q^{\epsilon}$ and $p^{\epsilon}$ :

$$
\begin{aligned}
& u^{\epsilon}(x, t)=u_{0}(x, x / \epsilon, t)+\epsilon u_{1}(x, x / \epsilon, t)+\epsilon^{2} u_{2}(x, x / \epsilon, t)+\ldots \\
& S^{\epsilon}(x, t)=S_{0}(x, x / \epsilon, t)+\epsilon S_{1}(x, x / \epsilon, t)+\epsilon^{2} S_{2}(x, x / \epsilon, t)+\ldots \\
& q^{\epsilon}(x, t)=q_{0}(x, x / \epsilon, t)+\epsilon q_{1}(x, x / \epsilon, t)+\epsilon^{2} q_{2}(x, x / \epsilon, t)+\ldots \\
& p^{\epsilon}(x, t)=p_{0}(x, x / \epsilon, t)+\epsilon p_{1}(x, x / \epsilon, t)+\epsilon^{2} p_{2}(x, x / \epsilon, t)+\ldots
\end{aligned}
$$

where $u_{k}(\cdot, y, \cdot), S_{k}(\cdot, y, \cdot), q_{k}(\cdot, y, \cdot)$ and $p_{k}(\cdot, y, \cdot)$ are 1-periodic in $y$. 
3.1. Preliminaries. In order to use the machinery of formal homogenization, we need to expand first of all $\nu^{\epsilon}$ in a power series in $\epsilon$. This can be done in terms of $S_{i}$ 's using (2.9). First we expand $\left|\nabla S^{\epsilon}\right|$ : using the well-known differentiation law [5]

$$
\nabla=\nabla_{x}+\frac{1}{\epsilon} \nabla_{y}
$$

the expansion for $S^{\epsilon}$ and the Taylor series for the square root, we obtain

$$
\left|\nabla S^{\epsilon}\right|=\frac{1}{\epsilon}\left|\nabla_{y} S_{0}\right|+(\ldots) .
$$

In the same fashion, we obtain

$$
\nu^{\epsilon}=\nu_{0}+\epsilon \nu_{1}+\epsilon^{2}(\ldots)
$$

where

$$
\nu_{0}=\frac{\nabla_{y} S_{0}}{\left|\nabla_{y} S_{0}\right|}
$$

and

$$
\nu_{1}=\frac{\nabla_{x} S_{0}+\nabla_{y} S_{1}}{\left|\nabla_{y} S_{0}\right|}-\frac{\left(\nabla_{x} S_{0} \cdot \nabla_{y} S_{0}+\nabla_{y} S_{0} \cdot \nabla_{y} S_{1}\right)}{\left|\nabla_{y} S_{0}\right|^{2}} \frac{\nabla_{y} S_{0}}{\left|\nabla_{y} S_{0}\right|}
$$

If we introduce $\tau_{0}=\nu_{0}^{\perp}$, we can write

$$
\nu_{1}=\tau_{0} \frac{\tau_{0} \cdot\left(\nabla_{x} S_{0}+\nabla_{y} S_{1}\right)}{\left|\nabla_{y} S_{0}\right|}
$$

Now we focus on the boundary conditions at the moving boundary $\Gamma^{\epsilon}(x, t)$. In order to obtain boundary conditions, we need to substitute the expansions for $u^{\epsilon}, q^{\epsilon}$ and $\nu^{\epsilon}$ in the boundary conditions (2.4). This is not so straight forward as it may seem, since the boundary conditions (2.4) are enforced at $\Gamma^{\epsilon}(t)$, i.e. at every $x$ where $S^{\epsilon}(x, t)=0$. For the formulation of the upscaled model it would be convenient to have a boundary condition enforced at $\Gamma_{0}(x, t):=\left\{y \mid S_{0}(x, y, t)=0\right\}$. To obtain such an equation, we suppose that we can parametrize the part of the boundary $\Gamma_{i j}^{\epsilon}(t)$ that surrounds the sphere $B_{i j}$ with $k^{\epsilon}(s, t)$, so that holds

$$
S^{\epsilon}\left(k^{\epsilon}(s, t), t\right)=0, \quad \text { for all } t \geq 0,
$$

and we assume that we can expand $k^{\epsilon}(s, t)$ using the formal asymptotic expansion

$$
k^{\epsilon}(s, t)=x+\epsilon k_{0}(s, t)+\epsilon^{2} k_{1}(s, t)+\epsilon^{3}(\ldots),
$$

with $x=(\epsilon i, \epsilon j)$. Using the expansion for $S^{\epsilon}$, the periodicity of $S_{i}$ in $y$, and the Taylor series for $S_{0}$ and $S_{1}$ around $\left(x, k_{0}\right)$, we obtain

$$
S_{0}\left(x, k_{0}, t\right)+\epsilon\left(S_{1}\left(x, k_{0}, t\right)+k_{0} \cdot \nabla_{x} S_{0}\left(x, k_{0}, t\right)+k_{1} \cdot \nabla_{y} S_{0}\left(x, k_{0}, t\right)\right)+\epsilon^{2}(\ldots)=0 .
$$


Collecting terms with the same order of $\epsilon$, we see that $k_{0}(s, t)$ parametrizes locally the zero level set of $S_{0}$ :

$$
S_{0}\left(x, k_{0}, t\right)=0
$$

For $k_{1}$, we have the equation

$$
S_{1}\left(x, k_{0}, t\right)+k_{0} \cdot \nabla_{x} S_{0}\left(x, k_{0}, t\right)+k_{1} \cdot \nabla_{y} S_{0}\left(x, k_{0}, t\right)=0 .
$$

It suffices to seek for $k_{1}$ that is aligned with $\nu_{0}$, so that we write

$$
\left.k_{1}(s, t)=\lambda(s, t)\right) \nu_{0}(s, t)=\lambda \frac{\nabla_{y} S_{0}}{\left|\nabla_{y} S_{0}\right|}
$$

where, using (3.4), $\lambda$ is given by

$$
\lambda=-\frac{S_{1}}{\left|\nabla_{y} S_{0}\right|}-\frac{k_{0} \cdot \nabla_{x} S_{0}}{\left|\nabla_{y} S_{0}\right|} .
$$

Let us consider an abstract boundary condition that can be written in the form

$$
K(x, x / \epsilon, t)=0, \quad \text { for } \quad x \in \Gamma^{\epsilon}(t) .
$$

Using the expansion (3.3) and the Taylor series for $K$ around $\left(x, k_{0}\right)$, we obtain

$$
\begin{aligned}
K\left(x, k_{0}, t\right)+ & \epsilon\left(k_{0} \cdot \nabla_{x} K\left(x, k_{0}, t\right)+k_{1} \cdot \nabla_{y} K\left(x, k_{0}, t\right)\right) \\
& +\frac{\epsilon^{2}}{2}\left(k_{0}, k_{1}\right) \cdot\left(\mathcal{D}^{2} K\left(x, k_{0}, t\right)\right)\left(k_{0}, k_{1}\right)+\epsilon^{3}(\ldots)=0,
\end{aligned}
$$

where $\mathcal{D}^{2} K$ denotes the Hessian of $K$. Substituting the expression (3.5), we can restate (3.7) in the following way:

$$
\begin{aligned}
K(x, y, t)+ & \epsilon\left(y \cdot \nabla_{x} K(x, y, t)+\lambda \nu_{0} \cdot \nabla_{y} K(x, y, t)\right) \\
+ & \frac{\epsilon^{2}}{2}\left(y, \lambda \nu_{0}\right) \cdot\left(\mathcal{D}^{2} K(x, y, t)\right)\left(y, \lambda \nu_{0}\right)+\epsilon^{3}(\ldots)=0, \text { for } y \in \Gamma_{0}(x, t) .
\end{aligned}
$$

Furthermore we will need the following technical lemmas in the subsequent sections.

LEMMA 3.1. Let $g(x, y, t)$ be a scalar function such that $g(x, y, t)=0$ for $y \in \Gamma_{0}(x, t)$, $x \in \Omega$ and $t \geq 0$, then it holds that

$$
\nabla_{x} g=\frac{\nu_{0} \cdot \nabla_{y} g}{\left|\nabla_{y} S_{0}\right|} \nabla_{x} S_{0}, \text { for } x \in \Omega, t \geq 0, y \in \Gamma_{0}(x, t) .
$$

Proof. Let $k_{0}(s, x)$ for $0 \leq s \leq 1$ parametrize $\Gamma_{0}(x, t)$. This means that $S_{0}\left(x, k_{0}(s, x)\right)=$ 0 , and since $g=0$ on $\Gamma_{0}(x, t)$, we also have $g(x, k(s, x))=0$. By differentiation with respect to $x_{i}$, for $i=1,2$, we obtain

$$
\begin{aligned}
\partial_{x_{i}} S_{0} & =-\nabla_{y} S_{0} \cdot \partial_{x_{i}} k_{0}=-\left|\nabla_{y} S_{0}\right| \nu_{0} \cdot \partial_{x_{i}} k_{0} \\
\partial_{x_{i}} g & =-\nabla_{y} g \cdot \partial_{x_{i}} k_{0}=-\left|\nabla_{y} S_{0}\right| \nu_{0} \cdot \partial_{x_{i}} k_{0}
\end{aligned}
$$


Since $g$ is zero on $\Gamma_{0}$, its gradient is parallel to $\nu_{0}$ and thus $\nabla_{y} g=\left(\nu_{0} \cdot \nabla_{y} g\right) \nu_{0}$, so that we obtain

$$
\partial_{x_{i}} g=-\nabla_{y} g \cdot \partial_{x_{i}} k_{0}=-\left(\nu_{0} \cdot \nabla_{y} g\right)\left(\nu_{0} \cdot \partial_{x_{i}} k_{0}\right)=\frac{\nu_{0} \cdot \nabla_{y} g}{\left|\nabla_{y} S_{0}\right|} \partial_{x_{i}} S_{0}
$$

This proofs the lemma.

LEMMA 3.2. Let $F(x, y, t)$ be a vector valued function such that $\nabla_{y} \cdot F(x, y, t)=0$ on $Y_{0}(x, t):=\left\{y \mid S_{0}(x, y, t)<0\right\}$ and $\nu_{0} \cdot F(x, y, t)=0$ on $\Gamma_{0}(x, t)$ for $x \in \Omega$ and $t \geq 0$. Then it holds that

$$
\int_{\Gamma^{0}(x, t)} \frac{\tau_{0} \cdot \nabla_{y} S_{1}}{\left|\nabla_{y} S_{0}\right|} \tau_{0} \cdot F-\frac{S_{1}}{\left|\nabla_{y} S_{0}\right|} \nu_{0} \cdot \nabla_{y}\left(\nu^{0} \cdot F\right) d \sigma=0, \text { for } x \in \Omega, t \geq 0 .
$$

Proof. We study variations of the integral

$$
I_{\delta}=\int_{Y^{\delta}(x, t)} \nabla_{y} \cdot F(x, y, t) d y,
$$

with respect to variation of the domain of integration. Here $Y^{\delta}(x, t)$ is the region where $S_{0}(x, y, t)+\delta S_{1}(x, y, t)<0$. In order to make sure that $Y^{\delta}$ is included in $Y_{0}$ on which $F$ is defined, we split $S_{1}$ into its positive and negative part, $S_{1}=\left[S_{1}\right]_{+}-\left[S_{1}\right]_{-}$, and define $Y_{+}^{\delta}:=\left\{y \mid S_{0}+\delta\left[S_{1}\right]_{+}<0\right\}$ and $Y_{-}^{\delta}:=\left\{y \mid S_{0}+\delta\left[S_{1}\right]_{-}<0\right\}$ which are both, for positive $\delta$, contained in $Y_{0}$. The right derivative with respect to $\delta$ of the integrals

$$
\int_{Y_{+}^{\delta}} \nabla_{y} \cdot F(x, y, t) d y \text { and } \int_{Y_{-}^{\delta}} \nabla_{y} \cdot F(x, y, t) d y
$$

equals zero due to the fact that $\nabla_{y} \cdot F=0$ on $Y_{0}(x, t)$. Suppose now that $k_{+}(s, \delta)$ parametrizes $\Gamma_{+}^{\delta}:=\partial Y_{+}^{\delta}$ so that $S_{0}\left(x, k_{+}(s, \delta), t\right)+\delta\left[S_{1}\right]_{+}\left(x, k_{+}(s, \delta), t\right)=0$. By differentiation with respect to $\delta$ we obtain

$$
\nabla_{y} S_{0} \partial_{\delta} k_{+}+\left[S_{1}\right]_{+}+\delta \nabla_{y}\left[S_{1}\right]_{+} \partial_{\delta} k_{+}=0, \text { for } y \in \Gamma_{+}^{\delta} .
$$

Thus for $\delta=0$, we have $\nabla_{y} S_{0} \partial_{\delta} k_{+}=-\left[S_{1}\right]_{+}$. Now we compute, where all derivatives with respect to $\delta$ are right derivatives:

$$
\begin{aligned}
0= & \left.\partial_{\delta} \int_{Y_{+}^{\delta}} \nabla_{y} \cdot F d y\right|_{\delta=0}=\left.\partial_{\delta} \int_{\Gamma_{+}^{\delta}} \nu^{\delta} \cdot F d \sigma\right|_{\delta=0} \\
= & \left.\partial_{\delta} \int_{0}^{1} \nu^{\delta} \cdot F\left(x, k_{+}(s, \delta), t\right)\left|\partial_{s} k_{+}(s, \delta)\right| d s\right|_{\delta=0} \\
= & \int_{0}^{1}\left(\left.\partial_{\delta} \nu^{\delta}\right|_{\delta=0} \cdot F+\left.\nabla_{y}\left(\nu^{0} \cdot F\right) \cdot \partial_{\delta} k_{+}\right|_{\delta=0}\right)\left|\partial_{s} k_{+}(s, 0)\right| d s+ \\
& \left.\int_{0}^{1} \nu^{0} \cdot F \partial_{\delta}\left(\left|\partial_{s} k_{+}(s, \delta)\right|\right)\right|_{\delta=0} d s \\
= & \int_{\Gamma^{0}} \frac{\tau_{0} \cdot \nabla_{y}\left[S_{1}\right]_{+}}{\left|\nabla_{y} S_{0}\right|} \tau_{0} \cdot F-\frac{\left[S_{1}\right]_{+}}{\left|\nabla_{y} S_{0}\right|} \nu_{0} \cdot \nabla_{y}\left(\nu^{0} \cdot F\right) d \sigma .
\end{aligned}
$$

For the last equality, we use again that $\nu^{0} \cdot F=0$ on $\Gamma^{0}$ so that $\nabla_{y}\left(\nu^{0} \cdot F\right)=\left(\nu_{0} \cdot \nabla_{y}\left(\nu_{0}\right.\right.$. $F)) \nu_{0}$. We also use a formula similar to (3.2) for the derivative of $\nu^{\delta}$ with respect to $\delta$. For the remaining of the proof, we perform the above computation also for the negative part $\left[S_{1}\right]_{-}$, and then subtract the results to complete the proof. 
3.2. Level set equation. Before we procede with the substitution of the formal expansions into the model equations, we will first regularize the set-valued Heaviside graph. This means that we replace $H$ in $\left(2.3_{2}\right)$ with $H_{\delta}$ which is defined, for small $\delta>0$, by

$$
H_{\delta}(d):= \begin{cases}0 & \text { if } d<0 \\ d / \delta & \text { if } d \in[0, \delta] \\ 1, & \text { if } d>\delta\end{cases}
$$

Furthermore, we introduce the notation

$$
f_{\delta}\left(u^{\epsilon}, y\right):=k\left(r\left(u^{\epsilon}\right)-H_{\delta}\left(\operatorname{dist}\left(\epsilon y, B^{\epsilon}\right)\right)\right),
$$

where $f_{\delta}(\cdot, y)$ is 1 -periodic in $y$. We replace the equations $(2.3)$ and (2.4) with

$$
\begin{aligned}
& S_{t}^{\epsilon}=\epsilon f_{\delta}\left(u^{\epsilon}, x / \epsilon\right)\left|\nabla S^{\epsilon}\right|, \quad \text { in } \Omega, \\
& \left\{\begin{array}{l}
\nu^{\epsilon} \cdot\left(D \nabla u^{\epsilon}-q^{\epsilon} u^{\epsilon}\right)=-\epsilon f_{\delta}\left(u^{\epsilon}, x / \epsilon\right)\left(\rho-u^{\epsilon}\right), \\
q^{\epsilon}=-\epsilon K f_{\delta}\left(u^{\epsilon}, x / \epsilon\right) \nu^{\epsilon},
\end{array}\right.
\end{aligned}
$$

Since $r$ and $H_{\delta}$ are both Lipschitz, we now write,

$$
f_{\delta}\left(u^{\epsilon}, y\right)=f_{\delta}\left(u_{0}, y\right)+\epsilon(\ldots) \text {. }
$$

Substituting (3.1) in $\left(2.3_{1}\right)$ and using the above expression, we obtain the equation

$$
\partial_{t} S_{0}-f_{\delta}\left(u_{0}, y\right)\left|\nabla_{y} S_{0}\right|+\epsilon(\ldots)=0
$$

Only keeping terms independent of $\epsilon$, and letting $\delta \rightarrow 0$, we obtain (see e.g., [15], Theorem 2.21)

$$
\partial_{t} S_{0}=f\left(u_{0}, y\right)\left|\nabla_{y} S_{0}\right|
$$

where $f\left(u_{0}, y\right)=k\left(r\left(u_{0}\right)-w\right)$, with $w \in H\left(\operatorname{dist}\left(\epsilon y, B^{\epsilon}\right)\right)$. (Note that this last expression does not depend on $\epsilon$.)

3.3. Convection-diffusion equation. When we substitute the asymptotic expansion for $u^{\epsilon}$ in $\left(2.2_{1}\right)$, we obtain

$$
\begin{aligned}
\partial_{t} u_{0}= & \frac{1}{\epsilon^{2}} D \Delta_{y} u_{0}+\frac{1}{\epsilon}\left(\nabla_{y} \cdot F+\nabla_{x} \cdot\left(D \nabla_{y} u_{0}\right)\right) \\
& +\nabla_{y} \cdot\left(D\left(\nabla_{y} u_{2}+\nabla_{x} u_{1}\right)-q_{1} u_{0}-q_{0} u_{1}\right)+\nabla_{x} \cdot F \\
& +\epsilon(\ldots),
\end{aligned}
$$

where $F=D\left(\nabla_{x} u_{0}+\nabla_{y} u_{1}\right)-q_{0} u_{0}$. Now we will first expand (2.4 $)$, using the expansions for $u^{\epsilon}, \nu^{\epsilon}$ and the Taylor series of $f$ around $u_{0}$ :

$$
\begin{aligned}
0 & =\nu^{\epsilon} \cdot\left(D \nabla u^{\epsilon}-q^{\epsilon} u^{\epsilon}\right)-f\left(u^{\epsilon}, y\right)\left(\rho-u^{\epsilon}\right) \\
& =\frac{1}{\epsilon} \nu_{0} \cdot\left(D \nabla_{y} u_{0}\right)+\nu_{0} \cdot F+\nu_{1} \cdot\left(D \nabla_{y} u_{0}\right) \\
& +\epsilon\left(\nu_{0} \cdot\left(D\left(\nabla_{x} u_{1}+\nabla_{y} u_{2}\right)-q_{1} u_{0}-q_{0} u_{1}\right)+\nu_{1} \cdot F+\nu_{2} \cdot\left(D \nabla_{y} u_{0}\right)-f\left(u_{0}, y\right)\left(\rho-u_{0}\right)\right) \\
& +\epsilon^{2}(\ldots), \text { for } x \in \Gamma^{\epsilon}(t), y=\frac{x}{\epsilon},
\end{aligned}
$$


and next we substitute this expansion in (3.8), and thus obtain

$$
\begin{aligned}
0= & \frac{1}{\epsilon} \nu_{0} \cdot\left(D \nabla_{y} u_{0}\right) \\
+ & \nu_{0} \cdot F+\nu_{1} \cdot\left(D \nabla_{y} u_{0}\right)+y \cdot \nabla_{x}\left(\nu_{0} \cdot\left(D \nabla_{y} u_{0}\right)\right)+\lambda \nu_{0} \cdot \nabla_{y}\left(\nu_{0} \cdot\left(D \nabla_{y} u_{0}\right)\right) \\
+ & \epsilon\left(\nu_{0} \cdot\left(D\left(\nabla_{x} u_{1}+\nabla_{y} u_{2}\right)-q_{1} u_{0}-q_{0} u_{1}\right)+\nu_{1} \cdot F+\nu_{2} \cdot\left(D \nabla_{y} u_{0}\right)\right. \\
& -f\left(u_{0}\right)\left(\rho-u_{0}\right)+y \cdot \nabla_{x}\left(\nu_{0} \cdot F+\nu_{1} \cdot\left(D \nabla_{y} u_{0}\right)\right)+\lambda \nu_{0} \cdot \nabla_{y}\left(\nu_{0} \cdot F+\nu_{1} \cdot\left(D \nabla_{y} u_{0}\right)\right) \\
& \left.+\frac{1}{2}\left(y, \lambda \nu_{0}\right) \cdot\left(\mathcal{D}^{2}\left(\nu_{0} \cdot\left(D \nabla_{y} u_{0}\right)\right)\right)\left(y, \lambda \nu_{0}\right)\right) \\
+ & \epsilon^{2}(\ldots), \text { for } y \in \Gamma_{0}(x, t) .
\end{aligned}
$$

Now we collect the $\epsilon^{-2}$-term from (3.14) and the $\epsilon^{-1}$-term from (3.15) and obtain for $u_{0}$ the equations

$$
\begin{cases}\Delta_{y} u_{0}=0 & \text { in } Y_{0}(x, t) \\ \nu_{0} \cdot \nabla_{y} u_{0}=0 & \text { on } \Gamma_{0}(x, t) \\ \text { periodicity in } y, & \end{cases}
$$

where $Y_{0}(x, t)=\left\{y \mid S_{0}(x, y, t)<0\right\}$. This means that $u_{0}$ is determined up to a constant and does not depend on $y$, so that $\nabla_{y} u_{0}=0$. Collecting the $\epsilon^{-1}$ terms from (3.14), the $\epsilon^{0}$-terms from (3.15), and using that $\nabla_{y} u_{0}=0$, we get for $u_{1}$ the equations

$$
\begin{cases}\nabla_{y} \cdot\left(D \nabla_{y} u_{1}-q_{0} u_{0}\right)=0 & \text { in } Y_{0}(x, t) \\ \nu_{0} \cdot\left(\nabla_{x} u_{0}+\nabla_{y} u_{1}-q_{0} u_{0}\right)=0 & \text { on } \Gamma_{0}(x, t) \\ \text { periodicity in } y & \end{cases}
$$

Next we collect the $\epsilon^{0}$-terms from (3.14) and the $\epsilon^{1}$-terms from (3.15) and obtain

$$
\begin{cases}\partial_{t} u_{0}=\nabla_{y} \cdot\left(D\left(\nabla_{y} u_{2}+\nabla_{x} u_{1}\right)-q_{1} u_{0}-q_{0} u_{1}\right)+\nabla_{x} \cdot F & \text { in } Y_{0}(x, t), \\ \nu_{0} \cdot\left(D\left(\nabla_{x} u_{1}+\nabla_{y} u_{2}\right)-q_{1} u_{0}-q_{0} u_{1}\right)=-\nu_{1} \cdot F & \\ \quad \quad+f\left(u_{0}\right)\left(\rho-u_{0}\right)-y \cdot \nabla_{x}\left(\nu_{0} \cdot F\right)-\lambda \nu_{0} \cdot \nabla_{y}\left(\nu_{0} \cdot F\right) & \text { on } \Gamma_{0}(x, t) \\ \text { periodicity in } y . & \end{cases}
$$

Integrating $\left(3.18_{1}\right)$ over $Y_{0}(x, t)$ and using the boundary condition $\left(3.18_{2}\right)$ gives

$$
\begin{aligned}
\left|Y_{0}(x, t)\right| \partial_{t} u_{0}= & \int_{Y_{0}(x, t)} \nabla_{y} \cdot\left(D\left(\nabla_{x} u_{1}+\nabla_{y} u_{2}\right)-q_{1} u_{0}-q_{0} u_{1}\right) d y+\int_{Y_{0}(x, t)} \nabla_{x} \cdot F d y \\
= & \int_{\Gamma_{0}(x, t)}-\nu_{1} \cdot F+f\left(u_{0}\right)\left(\rho-u_{0}\right)-y \cdot \nabla_{x}\left(\nu_{0} \cdot F\right)-\lambda \nu_{0} \cdot \nabla_{y}\left(\nu_{0} \cdot F\right) d \sigma \\
& +\nabla_{x} \cdot \int_{Y_{0}(x, t)} F d y+\int_{\Gamma_{0}(x, t)} \frac{\nabla_{x} S_{0}}{\left|\nabla_{y} S_{0}\right|} \cdot F d \sigma .
\end{aligned}
$$

This can be rewritten, using (3.2) and the boundary condition from (3.17), as

$$
\partial_{t}\left(\left|Y_{0}(x, t)\right| u_{0}\right)=\nabla_{x} \cdot \int_{Y_{0}(x, t)}\left(D\left(\nabla_{y} u_{1}+\nabla_{x} u_{0}\right)-q_{0} u_{0}\right) d y+\left|\Gamma_{0}(x, t)\right| f\left(u_{0}\right) \rho-I_{1}-I_{2},
$$


where

$$
\begin{aligned}
& I_{1}=\int_{\Gamma_{0}(x, t)} y \cdot \nabla_{x} g-\frac{y \cdot \nabla_{x} S_{0}}{\left|\nabla_{y} S_{0}\right|} \nu_{0} \cdot \nabla_{y} g d \sigma \\
& I_{2}=\int_{\Gamma_{0}(x, t)} \frac{\tau_{0} \cdot \nabla_{y} S_{1}}{\left|\nabla_{y} S_{0}\right|} \tau_{0} \cdot F-\frac{S_{1}}{\left|\nabla_{y} S_{0}\right|} \nu_{0} \cdot \nabla_{y}\left(\nu^{0} \cdot F\right) d \sigma
\end{aligned}
$$

with $g=\nu_{0} \cdot F$, and where we have used (3.6). The boundary condition $\left(3.17_{2}\right)$ gives us $g(x, y, t)=0$ for $y \in \Gamma_{0}(x, t)$, and now we invoke Lemma 3.1 to obtain $\nabla_{x} g=\frac{\nu_{0} \cdot \nabla_{y} g}{\left|\nabla_{y} S_{0}\right|} \nabla_{x} S_{0}$, so that $I_{1}=0$. For the integral $I_{2}$ we invoke Lemma 3.2 to obtain $I_{2}=0$.

3.4. Stokes equation. Substituting the asymptotic expansions for $q^{\epsilon}$ and $p^{\epsilon}$ in $\left(2.2_{2,3}\right)$, we obtain

$$
\begin{aligned}
& \mu \Delta_{y} q_{0}=\frac{1}{\epsilon} \nabla_{y} p_{0}+\nabla_{y} p_{1}+\nabla_{x} p_{0}+\epsilon(\ldots), \\
& \frac{1}{\epsilon} \nabla_{y} \cdot q_{0}+\nabla_{x} \cdot q_{0}+\nabla_{y} \cdot q_{1}+\epsilon(\ldots)=0,
\end{aligned}
$$

and substituting the expansion in the boundary condition $\left(2.4_{2}\right)$, and using (3.8), gives

$$
q_{0}+\epsilon\left(q_{1}-K f\left(u_{0}\right) \nu_{0}+\left(\nabla_{x} q_{0}\right)^{T} y+\lambda\left(\nabla_{y} q_{0}\right)^{T} \nu_{0}\right)+\epsilon^{2}(\ldots)=0, \text { for } y \in \Gamma_{0}(x, t) .
$$

The $\epsilon^{-1}$-term in (3.19) states $\nabla_{y} p_{0}=0$, so that we conclude that $p_{0}$ is independent of $y$. Furthermore, we obtain, after collecting $\epsilon^{0}$-terms from (3.19) and (3.21) and $\epsilon^{-1}$-terms from (3.20), the equations for $q_{0}$ and $p_{1}$ :

$$
\begin{cases}\mu \Delta_{y} q_{0}=\nabla_{y} p_{1}+\nabla_{x} p_{0} & \text { in } Y_{0}(x, t) \\ \nabla_{y} \cdot q_{0}=0 & \text { in } Y_{0}(x, t) \\ q_{0}=0 & \text { on } \Gamma_{0}(x, t) \\ \text { periodicity in } y & \end{cases}
$$

These equations determine the averaged velocity field given by

$$
\bar{q}(x, t)=\int_{Y_{0}(x, t)} q_{0}(x, y, t) d y .
$$

Now we compute the divergence of $\bar{q}$ (where we use the $\epsilon^{0}$-terms from (3.20))

$$
\begin{aligned}
\nabla_{x} \cdot \bar{q} & =\nabla_{x} \cdot \int_{Y_{0}(x, t)} q_{0} d y=\int_{Y_{0}(x, t)} \nabla_{x} \cdot q_{0} d y-\int_{\Gamma_{0}(x, t)} \frac{\nabla_{x} S_{0}}{\left|\nabla_{y} S_{0}\right|} \cdot q_{0} d \sigma \\
& =-\int_{Y(x, t)} \nabla_{y} \cdot q_{1} d y=-\int_{\Gamma_{0}(x, t)} \nu_{0} \cdot q_{1} d \sigma \\
& =\int_{\Gamma_{0}(x, t)} K f\left(u_{0}\right)-\nu_{0} \cdot\left(\left(\nabla_{x} q_{0}\right)^{T} y+\lambda\left(\nabla_{y} q_{0}\right)^{T} \nu_{0}\right) d \sigma \\
& =\left|\Gamma_{0}(x, t)\right| K f\left(u_{0}\right)-I_{1}-I_{2},
\end{aligned}
$$


with

$$
\begin{aligned}
& I_{1}=\int_{\Gamma_{0}(x, t)} \nu_{0} \cdot\left(\left(\nabla_{x} q_{0}\right)^{T} y-\frac{y \cdot \nabla_{x} S_{0}}{\left|\nabla_{y} S_{0}\right|}\left(\nabla_{y} q_{0}\right)^{T} \nu_{0}\right) d \sigma, \\
& I_{2}=-\int_{\Gamma_{0}(x, t)} \nu_{0} \cdot\left(\frac{S_{1}}{\left|\nabla_{y} S_{0}\right|}\left(\nabla_{y} q_{0}\right)^{T} \nu_{0}\right) d \sigma .
\end{aligned}
$$

We apply Lemma 3.1 to $g=\nu_{0} \cdot q_{0}$, and we obtain

$$
\nabla_{x}\left(\nu_{0} \cdot q_{0}\right)=\frac{\nu_{0} \cdot \nabla_{y}\left(\nu_{0} \cdot q_{0}\right)}{\left|\nabla_{y} S_{0}\right|} \nabla_{x} S_{0}, \quad \text { on } \Gamma_{0}(x, t) .
$$

Since $q_{0}=0$ on $\Gamma_{0}(x, t)$ it follows that $\left(\nabla_{x} q_{0}\right)^{T} \nu_{0}=\frac{\nu_{0} \cdot\left(\nabla_{y} q_{0}\right)^{T} \nu_{0}}{\left|\nabla_{y} S_{0}\right|} \nabla_{x} S_{0}$, so that $I_{1}=0$. Next we apply Lemma 3.2 to $q_{0}$, and consequently

$$
\int_{\Gamma^{0}} \frac{\tau_{0} \cdot \nabla_{y} S_{1}}{\left|\nabla_{y} S_{0}\right|} \tau_{0} \cdot q_{0}-\frac{S_{1}}{\left|\nabla_{y} S_{0}\right|} \nu_{0} \cdot \nabla_{y}\left(\nu^{0} \cdot q_{0}\right) d \sigma=0
$$

and again using $q_{0}=0$ on $\Gamma_{0}(x, t)$, it follows that $I_{2}=0$.

4. Upscaled equations and simplification. As usual, we write the solutions of equations (3.17) and (3.22) in terms of the solutions of the following two cell problems [5]

$$
\begin{cases}\Delta_{y} v_{j}=0 & \text { in } Y_{0}(x, t) \\ \nu_{0} \nabla_{y} v_{j}=-e_{j} & \text { on } \Gamma_{0}(x, t) \\ \text { periodicity in } y, & \end{cases}
$$

and

$$
\begin{cases}\Delta_{y} w_{j}=\nabla_{y} \pi_{j}+e_{j} & \text { in } Y_{0}(x, t) \\ \nabla_{y} \cdot w_{j}=0 & \text { in } Y_{0}(x, t) \\ w_{j}=0 & \text { on } \Gamma_{0}(x, t) \\ \text { periodicity in } y, & \end{cases}
$$

for $j=1,2$. This allows us to write the results of the formal homogenization procedure in the form of the following distributed microstructure model [5]

$$
\begin{aligned}
& \begin{cases}\partial_{t} S_{0}(x, y, t)+f\left(u_{0}(x, t), y\right)\left|\nabla_{y} S_{0}(x, y, t)\right|=0 & \text { for } y \in[0,1]^{2}, x \in \Omega \\
\partial_{t}\left(\left|Y_{0}(x, t)\right| u_{0}\right)=\nabla_{x} \cdot\left(D \mathcal{A}(x, t) \nabla_{x} u_{0}-\bar{q} u_{0}\right)+\left|\Gamma_{0}(x, t)\right| f\left(u_{0}\right) \rho & \text { for } x \in \Omega \\
\bar{q}=-\frac{1}{\mu} \mathcal{K}(x, t) \nabla_{x} p_{0} & \text { for } x \in \Omega \\
\nabla_{x} \cdot \bar{q}=\left|\Gamma_{0}(x, t)\right| K f\left(u_{0}\right) & \text { for } x \in \Omega\end{cases} \\
& \begin{cases}u_{0}(x, t)=u_{b}(x, t) \quad \text { for } x \in \Gamma \\
\bar{q}(x, t)=q_{b}(x, t) \quad \text { for } x \in \Gamma\end{cases} \\
& \left\{\begin{array}{l}
u_{0}(x, 0)=u_{I}(x) \\
S_{0}(x, y, 0)=S_{I}(x, y)
\end{array}\right.
\end{aligned}
$$


where the tensors $\mathcal{A}(x, t)=\left(a_{i j}\right)_{i, j}$ and $\mathcal{K}(x, t)=\left(k_{i j}\right)_{i, j}$ are given by

$$
a_{i j}=\int_{Y_{0}(x, t)} \delta_{i j}+\partial_{y_{i}} v_{j} d y
$$

and

$$
k_{i j}=\int_{Y_{0}(x, t)} w_{j i} d y,
$$

For computational purposes, the distributed microstructure model as introduced above is still not much more preferable as the original micro-scale model: it involves solving two cell problems for each $x$-gridpoint. This can be drastically reduced if we asume special initial micro-scale geometries. If we start with spherical grain boundaries, i.e., if the sets $\Gamma_{0}(x, 0)=\left\{y \mid S_{0}(x, y, 0)=0\right\}$ are a circles, then, since $f\left(u_{0}(x, t), y\right)$ is spherically symmetric in $y$ for each $x \in \Omega$, solutions of the level set equation $\left(4.3_{1}\right)$ will be such that the sets $\Gamma_{0}(x, t)$ will stay a circle for all $x \in \Omega$ and $t>0$. This means that in this case the equation $\left(4.3_{1}\right)$ can be reduced to

$$
\partial_{t} R(x, t)=f\left(u_{0}(x, t), R(x, t)\right)=k\left(r\left(u_{0}(x, t)\right)-w(x, t)\right.
$$

with $w \in H\left(R(x, t)-R_{\min }\right)$, and where $R(x, t)$ denotes the radius of $\Gamma_{0}(x, t)$. The other equations in (4.3) can also be simplified, and we obtain the system

$$
\begin{aligned}
& \left\{\begin{array}{l}
\partial_{t} R=k\left(r\left(u_{0}\right)-w\right) \\
w \in H\left(R-R_{\min }\right) \\
\partial_{t}\left(\left(1-\pi R^{2}\right) u_{0}\right)=\nabla_{x} \cdot\left(D \mathcal{A}(R) \nabla_{x} u_{0}-\bar{q} u_{0}\right)+2 \pi R k\left(r\left(u_{0}\right)-w\right) \rho \quad \text { in } \Omega \\
\bar{q}=-\frac{1}{\mu} \mathcal{K}(R) \nabla_{x} p_{0} \\
\nabla_{x} \cdot \bar{q}=2 \pi R K k\left(r\left(u_{0}\right)-w\right)
\end{array}\right. \\
& \left\{\begin{array}{l}
u_{0}(x, t)=u_{b}(x, t) \\
\bar{q}(x, t)=q_{b}(x, t)
\end{array}\right. \\
& \left\{\begin{array}{l}
u_{0}(x, 0)=u_{I}(x) \\
R(x, 0)=R_{I}(x)
\end{array}\right.
\end{aligned}
$$

where the tensors $\mathcal{A}(R(x, t))$ and $\mathcal{K}(R(x, t))$ are again determined by the solutions of the cell problems (4.1) and (4.2) which now only depend on $R(x, t)$, since $Y_{0}(x, t)$ is determined by $R(x, t)$. Note that the same reduction can also be obtained for different initial grain geometries: for ellipses the same would hold. The important condition is that solutions of the level set equation are in a certain sense shape invariant and can be parametrized with a single parameter.

5. Numerical experiments. In this section we compare numerical solutions of the original equations on the micro-scale with numerical solutions of the homogenized equations in order to see how well the homogenized equations approximate the averaged behavior of the original model. As a first test we compare the solution of the upscaled equations (4.7)-(4.9) to solutions of the original micro-scale model (2.2)-(2.6). The initial 


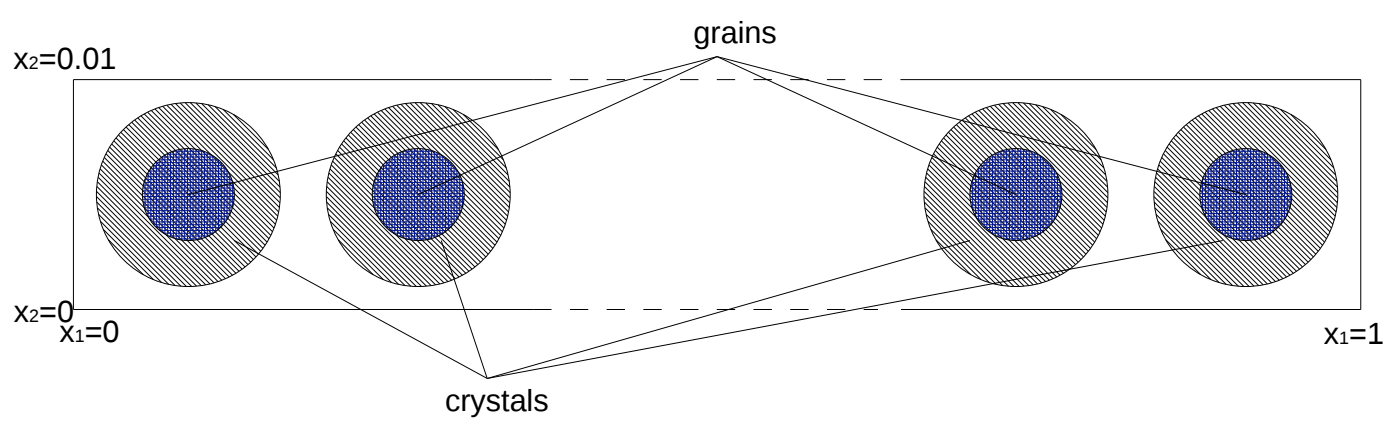

FIG. 5.1. The initial configuration for the micro-scale numerical experiments.

configuration for the micro-scale model is shown in Fig. 5.1: we consider a strip of length 1 and width 0.01 with a periodic array of inclusions. The inclusions are circles with initial radius 0.4 , and with midpoints $(x, y)=(0.005 \bmod 0.01,0.005)$ and consist of a grain of radius 0.2 and a layer of crystalline solid with thickness 0.2 . Thus initially we have a square periodic cell with hight and length $\epsilon=0.01$. Furthermore we consider periodicity in the "vertical" $x_{2}$-direction. This means that the domain $\Omega$ in the upscaled equations (4.7) reduces to the interval $(0,1)$.

For the micro-scale equations (2.2)-(2.6) we use the following parameter values

$$
\begin{array}{cc}
D=1, & \mu=1, \quad k=1, \\
\rho=2, & r(u)=u, \quad K=0,
\end{array}
$$

and we use the following boundary conditions in (2.5):

$$
\begin{aligned}
& \left\{\begin{array}{l}
u_{b}=0, \\
q_{b}=\left(\begin{array}{l}
1 \\
0
\end{array}\right), \quad \text { for } x_{1}=0,
\end{array}\right. \\
& \left\{\begin{array}{l}
\nu \cdot \nabla u=0, \\
p^{\epsilon} I+\frac{\mu}{2}\left(\nabla q^{\epsilon}+\left(\nabla q^{\epsilon}\right)^{T}\right)=0,
\end{array}\right. \\
& \text { periodicity in } x_{2} \text {, }
\end{aligned}
$$

and for the initial conditions in (2.6) we use

$$
u_{I} \equiv 1
$$

For the homogenized equations (4.7)-(4.9) this results in the same parameter values as in (5.1). The corresponding boundary conditions in (4.8) are

$$
\begin{aligned}
& \left\{\begin{array}{l}
u_{b}=0, \\
q_{b}=1,
\end{array} \text { for } x=0,\right. \\
& \partial_{x} u=0, \quad \text { for } x=1,
\end{aligned}
$$

and the corresponding initial conditions in (4.9) are

$$
u_{I} \equiv 1, \quad R_{I} \equiv 0.4
$$



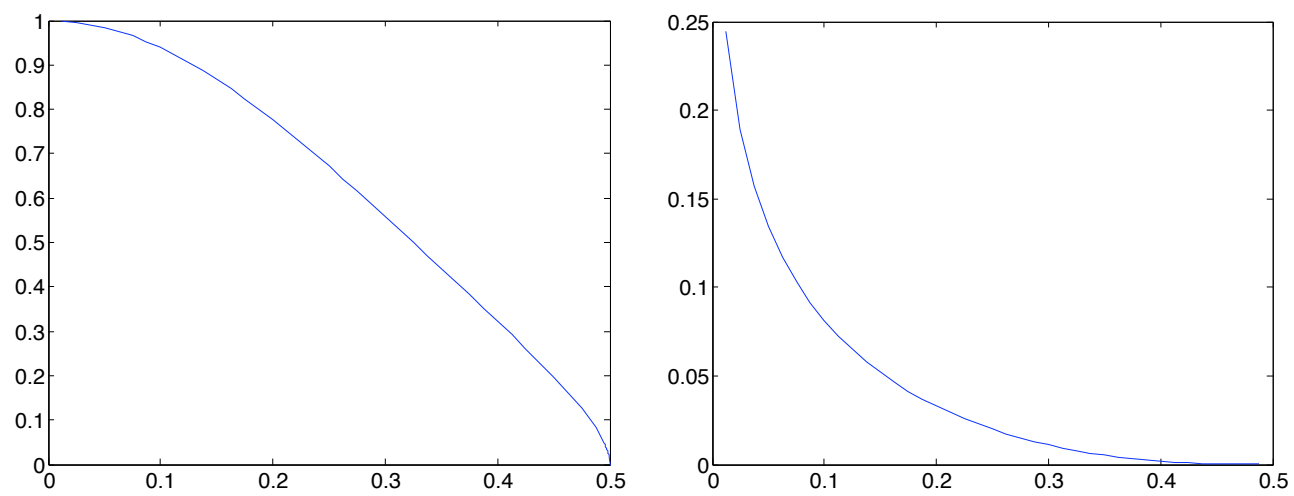

FIG. 5.2. The effective diffusion coefficient (left plot) and the effective permeability coefficient (right plot) as a function of the radius of the grain.

Note that, since $K=0$, we do not need to solve for $\bar{q}$. Due to $\left(4.7_{4}\right)$ we have that $\bar{q}=q_{b}$ for all $x \in[0,1]$. Also note that for a $1 \mathrm{D}$ setting such as the present one, we actually do not need to compute the effective permeability tensor $\mathcal{K}$ if we supply a boundary condition for $\bar{q}$. If we supply a pressure drop then we do need to know the tensor $\mathcal{K}$, as we do for computations in a $2 \mathrm{D}$ or higher dimensional setting.

5.1. Micro-scale models: Arbitrary Lagrangian Eulerian method. For the numerical solution solution of the micro-scale model we do not use the level set formulation, but we use the arbitrary Lagrangian Eulerian (ALE) method [2], where we use normal velocity as given in (2.1) for the computation of the deformation of the ALE mesh. The ALE method has been developed to solve partial differential equations on moving domains. In this method the partial time derivatives are expressed with respect to a fixed reference configuration. A map, called the ALE map, $\chi_{t}: \Omega_{0} \rightarrow \Omega(t)$, associates, at each time $t$, a point in the current computational domain $\Omega(t)$ to a point in the reference domain $\Omega_{0}$. In this way, the system of ordinary differential equations resulting after space discretization describes the evolution of the solutions along trajectories that are at all times contained in the computational domain. We use the ALE method as implemented in the COMSOL Multiphysics package [1], with Laplacian smoothing [2]. For more details on the ALE method we refer to $[1,2]$, and we also refer to [17] for the application of the ALE method to crystal dissolution. We discretize the equations (2.2)(2.6) using 16261 quadratic elements, resulting in 218961 unknowns. Furthermore we regularize the set-valued Heaviside graph $H$ for the numerical computations: we replace it by $H_{\delta}$ so that in the equation (2.1) for the normal velocity of the free boundary we have

$$
w^{\epsilon}(x, t)=H_{\delta}\left(\left|\left(x_{1}(\bmod 0.01), x_{2}\right)-(0.005,0.005)\right|-0.002\right),
$$

with $H_{\delta}$ as defined in (3.11) with $\delta=0.01$.

5.2. Upscaled model: effective diffusion and permeability tensors. In order to numerically approximate solutions of the upscaled equations (4.7), we need to compute the effective diffusion and permeability tensors as a function of the radius of the grain in the local cell problem. It is easily seen that in this case, due to symmetry, the tensors are in fact coefficients. We solved the cell problems (4.1) and (4.2) using a finite element 
discretization (quadratic elements) for various values of $R$. The values of $R$ are taken on a equidistant grid on the interval $[0.2,0.5]$ with 25 grid points. The number of elements ranged from 866 for $R=0.2$ to 450 for $R=0.4985$. Next we fitted these results with a fourth order polynomial in $(0.5-R)^{1 / 2}$ for the diffusion coefficients and a third order polynomial in $(0.5-R)$ for the permeability coefficient, which resulted in the following functions for $\mathcal{A}(R)$ and $\mathcal{K}(R)$ :

$$
\begin{aligned}
\mathcal{A}(R)= & 0.639879(0.5-R)^{1 / 2}+0.665634(0.5-R)+2.128861(0.5-R)^{3 / 2} \\
& -1.332304(0.5-R)^{2} \\
\mathcal{K}(R)= & 0.000207(0.5-R)+0.090013(0.5-R)^{2}+0.913720(0.5-R)^{3} .
\end{aligned}
$$

The choice for the form of these polynomials was based on the form of the graphs in Fig. 5.2 , where we have in both cases $\mathcal{A}(0.5)=0$ and $\mathcal{K}(0.5)=0$. (Note that we fitted these graphs only on the interval $[0.2,0.5]$, since the grain radii equal 0.2 in the scaled cellproblems.) With the fits given above, the maximal error in the computed points was in the order of $10^{-3}$ for the diffusion coefficient and in the order of $10^{-4}$ for the permeability coefficient.

For the numerical solution of the equations (4.7)-(4.9), we use a discretization of the interval $[0,1]$ into 240 quadratic elements, which results in a system with 722 unknowns. Furthermore, also for the homogenized equations we regularize the set-valued Heaviside graph $H$ so that in $\left(4.7_{2}\right)$ we obtain

$$
w(R)=H_{\delta}(R-0.2)
$$

with $\delta=0.01$.

5.3. Numerical results: micro-scale vs. upscaled equations. Initially, for $t=$ 0 , the system is in equilibrium: for the initial condition $u_{I} \equiv 1$ it holds that $r\left(u_{I}\right)=u_{I}=1$ so that there is no dissolution or precipitation. For positive $t$ there is, due to the boundary condition $u_{b}=0$ for $x_{1}=0$ in (5.2) and for $x=0$ in (5.5), transport of fluid with a low concentration of solute by diffusion and convection, and dissolution will start, first at the left end of the domain.

In Fig. 5.3 the ALE mesh is shown. The top plot depicts a part of the mesh at the left part of the domain in its initial state. The bottom plot depicts the mesh after all the crystalline solid is dissolved and the boundary of the mesh is located at the boundary of the grains.

In Fig. 5.4 the profiles of solutions of the micro-scale model and the homogenized model are compared for two different time instants, $t=0.05$ and $t=0.5$. In the left plot the concentrations $u^{\epsilon}$ and $u_{0}$ are given. For the micro-scale model the concentration $u^{\epsilon}$ (represented by the dots) is plotted along the line $y=0$. In the right plot the radius $R$ is plotted for the homogenized equations and an estimate of the radius of the inclusions for the micro-scale equations (again represented by the dots). We see that for $t=0.05$ only a small amount of crystalline solid is disolved, while for $t=0.5$ in approximately the left half of the domain already all the crystalline solid is disolved. We also see that the results for the micro-scale equations and the homogenized equations match very well. 

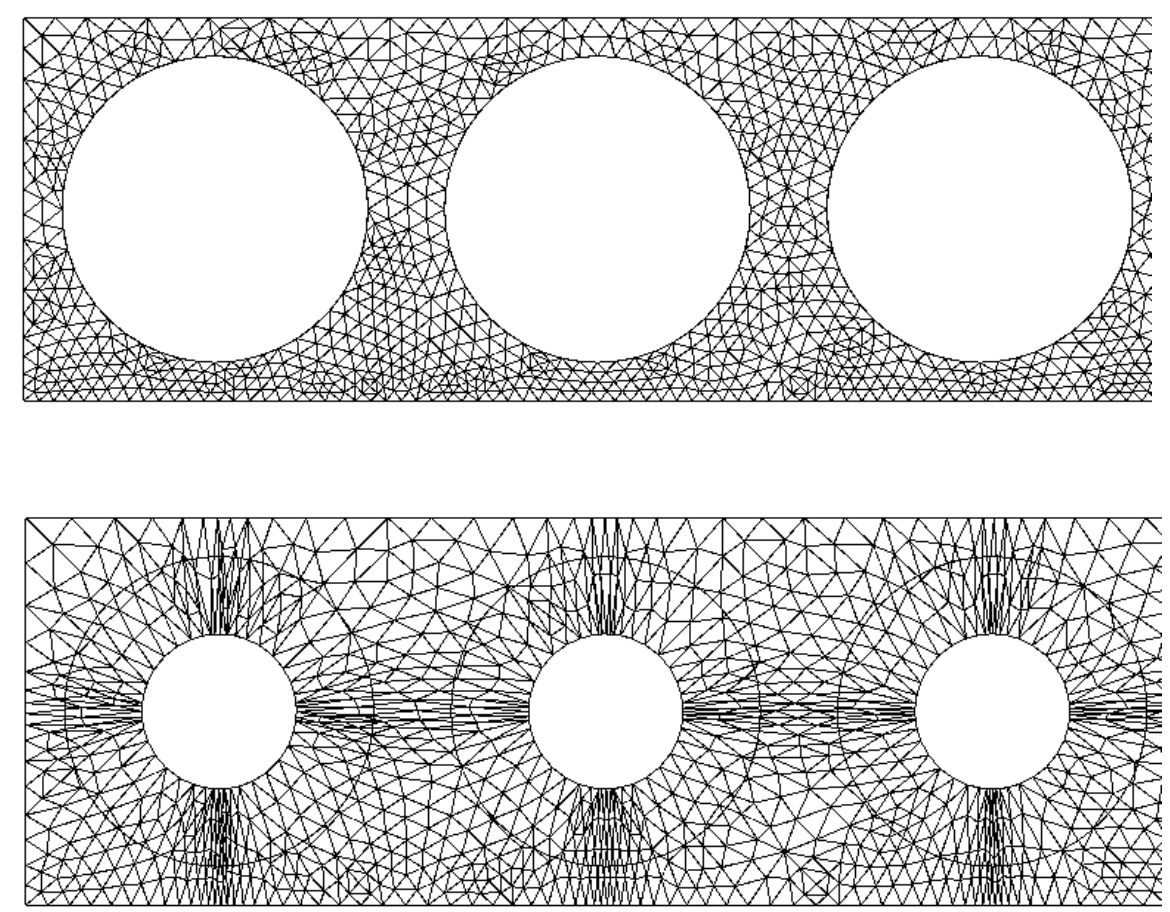

FIG. 5.3. The ALE mesh (for a part of the domain) for the initial configuration and for the dissolved configuration.
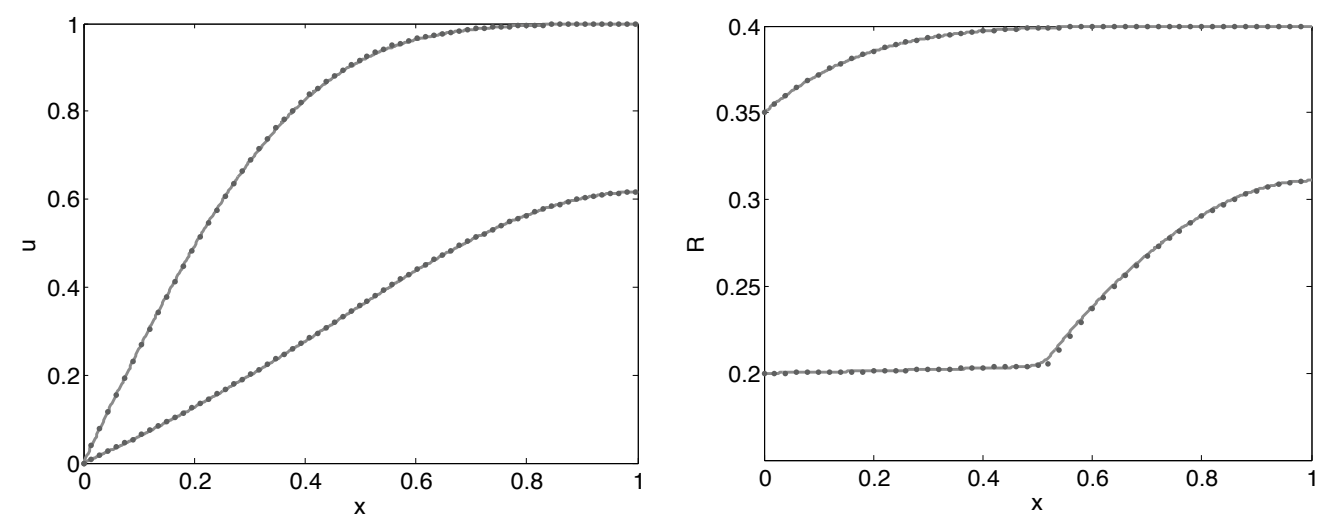

FIG. 5.4. Comparison of profiles for $t=0.05$ and $t=0.5$. The dots are the micro-scale model and the line is the homogenized model. In the left plot the concentration $u$ is depicted and in the right plot the radius $R$.

6. Conclusions. Using a level-set formulation of the free boundary arising in a model for crystal dissolution and precipitation, we are able to apply a formal homogenization procedure to obtain upscaled equations for crystal dissolution and precipitation in a porous medium. For general micro-scale geometries, the homogenized model is a distributed microstructure model. For circular initial inclusions the distributed microstructure model reduces to system of partial differential equations coupled with an ordinary differential equation. All the unknowns depend only on the slow variable. In order to investigate how well the upscaled equations describe the behavior of the micro-scale model, we perform numerical computations for a test problem. The numerical simulations show that for the 
test problem the solution of the homogenized equations agrees very well with the averaged solution of the micro-scale model.

Acknowledgements. The author wishes to thank I.S. Pop, C.J. van Duijn and M.A. Peletier for the discussions that helped to shape this work.

\section{REFERENCES}

[1] COMSOL Inc. http://www.comsol.com.

[2] J. Donea, A. Huerta, J.-Ph. Ponthot, And A. Rodríguez-Ferran, Arbitrary Lagrangian-Eulerian methods, in Encyclopedia of Computational Mechanics, Volume 1: Fundamentals, Chap. 14, E. Stein, R. De Borst, and T. J. R. Hughes, eds., John Wiley \& Sons, Ltd., 2004.

[3] R. Eymard, T. Gallouët, R. Herbin, D. Hilhorst, and M. Mainguy, Instantaneous and noninstantaneous dissolution: approximation by the finite volume method, in Actes du 30ème Congrès d'Analyse Numérique: CANum '98 (Arles, 1998), vol. 6 of ESAIM Proc., Soc. Math. Appl. Indust., Paris, 1999, pp. 41-55 (electronic).

[4] B. Faugeras, J. Pousin, and F. Fontvieille, An efficient numerical scheme for precise time integration of a diffusion-dissolution/precipitation chemical system, Math. Comp., 75 (2006), pp. 209-222 (electronic).

[5] U. Hornung, ed., Homogenization and porous media, vol. 6 of Interdisciplinary Applied Mathematics, Springer-Verlag, New York, 1997.

[6] U. Hornung, W. JÄGer, And A. Mikelić, Reactive transport through an array of cells with semi-permeable membranes, RAIRO Modél. Math. Anal. Numér., 28 (1994), pp. 59-94.

[7] P. Knabner, C. J. van Duijn, AND S. Hengst, An analysis of crystal dissolution fronts in flows though porous media. Part 1: Compatible boundary conditions, Adv. Water Res., 18 (1995), pp. 171-185.

[8] E. Maisse And J. Pousin, Diffusion and dissolution/precipitation in an open porous reactive medium, J. Comput. Appl. Math., 82 (1997), pp. 279-290. 7th ICCAM 96 Congress (Leuven).

[9] S. Osher AND R. Fedkiw, Level set methods and dynamic implicit surfaces, vol. 153 of Applied Mathematical Sciences, Springer-Verlag, New York, 2003.

[10] J. A. Sethian, Numerical algorithms for propagating interfaces: Hamilton-Jacobi equations and conservation laws, J. Differential Geom., 31 (1990), pp. 131-161.

[11] _ Level set methods, vol. 3 of Cambridge Monographs on Applied and Computational Mathematics, Cambridge University Press, Cambridge, 1996.

[12] A. Tartakovsky, T. Scheibe, G. Redden, P. Meakin, and Y. Fang, Smoothed particle hydrodynamics model for reactive transport and mineral precipitation, in Proceedings of the XVI International Conference on Computational Methods in Water Resources, Copenhagen, Denmark, June, 2006. http://proceedings.cmwr-xvi.org, P. J. Binning, P. K. Engesgaard, H. K. Dahle, G. F. Pinder, and W. G. Gray, eds.

[13] A. M. Tartakovsky, P. Meakin, T. D. Scheibe, and R. M. Eichler West, Simulations of reactive transport and precipitation with smoothed particle hydrodynamics, J. Comput. Phys., 222 (2007), pp. 654-672.

[14] C. J. van Duijn, L. A. Peletier, And R. J. Schotting, On the analysis of brine transport in porous media, European J. Appl. Math., 4 (1993), pp. 271-302.

[15] C. J. van Duijn And I. S. Pop, Crystal dissolution and precipitation in porous media: pore scale analysis, J. reine angew. Math., 577 (2004), pp. 171-211. 
[16] C. J. van Duijn And R. J. Schotting, Brine transport in porous media: on the use of von Mises and similarity transformations, Comput. Geosci., 2 (1998), pp. 125-149.

[17] T. L. VAn NOORDEn, Crystal precipitation and dissolution in a thin strip, tech. report, CASA report 30, Eindhoven University of Technology, 2007.

[18] T. L. van Noorden And I. S. Pop, A Stefan problem modelling dissolution and precipitation, IMA Journal of Applied Mathematics, (2007), pp. , doi: 10.1093/imamath/hxm060.

[19] T. L. VAn NoOrden, I. S. Pop, AND M. RÖGER, Crystal dissolution and precipitation in porous media: $L^{1}$-contraction and uniqueness, Discrete and Continuous Dynamical Systems Supplement, (2007), pp. 1013-1020. 